\title{
Jet-linked X-ray emission in radio-loud broad absorption line (BAL) quasars
}

\author{
M. Kunert-Bajraszewska ${ }^{1}$, K. Katarzyński ${ }^{1}$, A. Janiuk ${ }^{2}$, \\ and M. Cegłowski ${ }^{1}$ \\ ${ }^{1}$ Toruń Centre for Astronomy, N. Copernicus University, Gagarina 11, 87-100 Toruń, Poland \\ email: magda@astro.uni.torun.pl \\ ${ }^{2}$ Center for Theoretical Physics, PAS, Al.Lotnikow 32/46, 02-668 Warsaw, Poland
}

\begin{abstract}
We have applied theoretical models to explain spectral energy distribution (SED) of three radio-loud broad absorption line (BAL) quasars: an extended hybrid object PG 1004+130 and two compact sources $1045+352$ and $3 \mathrm{C} 270.1$. We calculate the emission from the very inner part of the sources which accounts for more than $90 \%$ of the observed X-ray radiation. In our analysis we consider a scenario in which the observed X-ray emission comes from the inverseCompton (IC) scattering inside a jet and from the accretion disk corona. The compact objects $1045+352$ and $3 \mathrm{C} 270.1$ are high-redshift quasars $(z=1.604$ and 1.532 respectively), with strong radio cores. We argue that in the case of these two sources a non-thermal, inverse-Compton emission from the innermost parts of the jet can explain a large fraction of the observed X-ray emission. The large scale object PG $1004+130$ with a peculiar radio morphology is a low-redshift $(\mathrm{z}=0.24)$, lobe-dominated BAL quasar with a weak radio core. In this case simulated inverseCompton X-ray emission of the jet is relatively low. However, the corona emission appears strong enough to explain the observed X-ray spectrum of this object.
\end{abstract}

Keywords. Physical data and processes: accretion, quasars: individual (1045+352, 3C270.1, PG 1004+130)

\section{Introduction}

According to the established paradigm, optical to UV emission of quasars results from accretion onto a super-massive black hole. The photons from optical to UV or even to soft $\mathrm{X}$-ray range are produced by the accretion disk. In addition, the corona that surrounds the disk may produce hard X-rays. The radio-loud quasars (those with radio loudness $R^{*} \geqslant 1$, Stocke et al. 1992) constitute a sub-class of quasars having strong radio emission linked to the presence of jets. Zamorani et al. (1981) demonstrated that radio-loud quasars are more X-ray luminous than radio-quiet quasars with comparable optical/UV luminosities. The excess of the X-ray emission increases with the radio loudness (Worrall et al. 1987). This may suggest a common physical background for both phenomena. However, it is still unclear which components and processes can contribute to the total X-ray emission. The non-thermal emission of the small-scale jet or the emission related to the accretion disk and hot corona, or both.

We focus here on BAL quasars, where the X-ray continuum probably contains both: disk-corona and small-scale jet emission (Miller et al. 2009, Kunert-Bajraszewska et al. 2009). 


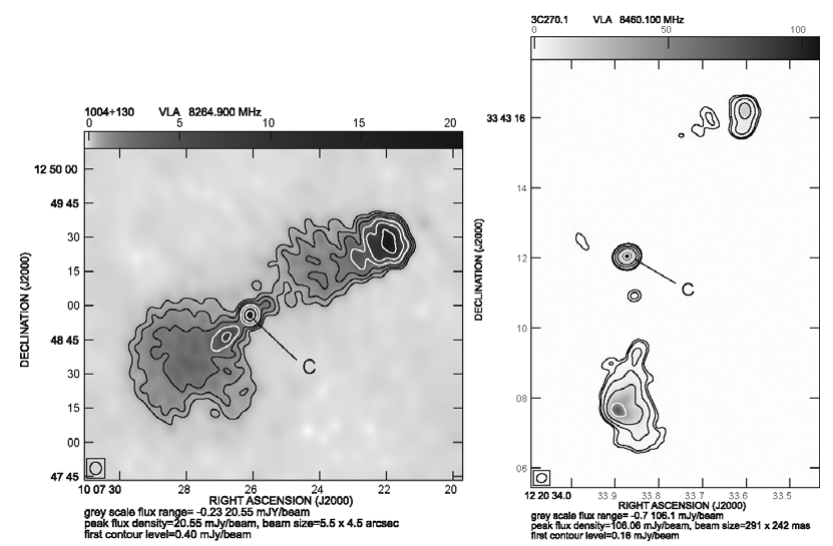

Figure 1. Radio $8 \mathrm{GHz}$ VLA images of PG $1004+130$ and 3C270.1. First contour level corresponds to $3 \sigma$. Indications: $\mathrm{C}$ - radio core.

\section{Discussion and Results}

Two of the studied quasars, PG 1004+130 and 3C270.1 are classified as large scale (linear size $>20 \mathrm{kpc}$ ), radio-loud AGNs. Their radio structures are well resolved with VLA (Fig. 1), showing central component - radio core and jets/lobes on both sides. As already discussed in the literature, the X-ray emission that comes from the outer parts of the radio jet and the hotspots, in the case of PG $1004+130$ and 3C270.1, could have either synchrotron or IC origin, but it accounts for $\sim 2 \%$ of the whole observed $\mathrm{X}$-ray emission. Most of the observed X-rays come from the very centre of the sources, where the radio cores (component $C$, Fig. 1) are probably connected with the small-scale jets. The detailed study of the third quasar, 1045+352, has been already presented in Kunert-Bajraszewska et al. (2009).

Broad-band spectra of PG 1004+130 and 3C270.1 are characterized by the strong radio emission, a peak in the IR range, and relatively strong UV-X-ray continuum. We used a simple synchrotron self-Compton model of the jet emission and also corona X-ray emission model to analyse central regions of both quasars. We argue that in the case of two core-dominated sources, $1045+352$ and 3C270.1, a non-thermal, inverse-Compton emission from the innermost parts of the radio jet can account for a large fraction of the observed X-ray emission. In the case of lobe dominated, weak radio core object PG 1004+130, the X-ray emission of the accretion disk and corona are needed to explain the observed X-ray emission. The central region of PG 1004+130 appears to be too weak to produce significant part of the observed X-ray emission.

\section{Acknowledgements}

This work was supported by the Polish Ministry of Science and Higher Education under grant UMO-2011/01/D/ST9/00378.

\section{References}

Kunert-Bajraszewska, M., Siemiginowska, A., Katarzyski, K., \& Janiuk, A., 2009, ApJ, 705, 1356

Miller, B. P. et al., 2009, ApJ, 702, 911

Stocke, J. T., Morris, S. L., Weymann, J. T., \& Foltz, C. B., 1992, ApJ, 396, 487

Worrall, D. M., Tananbaum, H., Giommi, P., \& Zamorani, G., 1987, ApJ, 313, 596

Zamorani, G. et al., 1981, ApJ, 245, 357 